\title{
THE FORMATION OF MILKY WAY-MASS DISK GALAXIES IN THE FIRST 500 MILLION YEARS OF A COLD DARK MATTER UNIVERSE
}

\author{
Yu Feng ${ }^{1}$, Tiziana Di Matteo ${ }^{2}$, Rupert Croft $^{2}$, Ananth Tenneti ${ }^{2}$, Simeon Bird $^{2}$, \\ Nicholas Battaglia ${ }^{3}$, and StePHen WiLKins ${ }^{4}$ \\ ${ }^{1}$ Berkeley Center for Cosmological Physics, University of California at Berkeley, Berkeley, CA 94720, USA; yfeng1@ berkeley.edu \\ ${ }^{2}$ McWilliams Center for Cosmology, Physics Department, Carnegie Mellon University, Pittsburgh, PA 15213, USA \\ ${ }^{3}$ Department of Astrophysical Sciences, Princeton University, Princeton, NJ 08544, USA \\ ${ }^{4}$ Astronomy Center, Department of Physics and Astronomy, University of Sussex, Brighton BN19QH, UK \\ Received 2015 April 24; accepted 2015 June 15; published 2015 July 20
}

\begin{abstract}
Whether or not among the myriad tiny protogalaxies there exists a population with similarities to present-day galaxies is an open question. We show, using BlueTides, the first hydrodynamic simulation large enough to resolve the relevant scales, that the first massive galaxies to form are predicted to have extensive rotationally supported disks. Although their morphology resembles in some ways Milky Way types seen at much lower redshifts, these high-redshift galaxies are smaller, denser, and richer in gas than their low-redshift counterparts. From a kinematic analysis of a statistical sample of 216 galaxies at redshift $z=8-10$, we have found that disk galaxies make up 70\% of the population of galaxies with stellar mass $10^{10} M_{\odot}$ or greater. Cold dark matter cosmology therefore makes specific predictions for the population of large galaxies 500 million years after the Big Bang. We argue that widefield satellite telescopes (e.g., WFIRST) will in the near future discover these first massive disk galaxies. The simplicity of their structure and formation history should make new tests of cosmology possible.
\end{abstract}

Key words: galaxies: high-redshift - galaxies: kinematics and dynamics - Galaxy: formation - hydrodynamics large-scale structure of universe

\section{INTRODUCTION}

The search for "primeval" galaxies and the understanding that will come from studying them has long been a guiding principle for much of extragalactic astrophysics and cosmological research. Theories for the formation of the first galaxies span early models of monolithic collapse of massive bodies (Eggen et al. 1962) to merger scenarios for the hierarchical formation of all structures (Press \& Schechter 1974). Disk galaxies are arguably those that have generated the most interest and were the focus of early theoretical work (Fall \& Efstathiou 1980). In the simplest galaxies, the structure of rotating disks should be directly linked to the angular momentum of the surrounding dark matter halo. Finding galaxies for which this is true, in the earliest environments possible, would enable powerful tests of the dynamics and gaseous and radiative processes involved in galaxy formation.

In current cosmological models, galaxies form from the gravitational collapse of small perturbations in the matter distribution (Silk \& Mamon 2012). This process involves both a hierarchy of merging structures and smooth accretion so that early galaxies are predicted to be morphologically irregular, clumpy, and compact (Cen \& Kimm 2014; Shimizu et al. 2014). This is supported by recent observational data on samples of galaxies at redshift $z=8$ and beyond (Oesch et al. 2010; Ono et al. 2013; Curtis-Lake et al. 2014). The volumes accessible to these studies, both computational and observational, however, are thousands of times smaller than those that will be probed by upcoming telescopes (Spergel et al. 2015).

The earliest that large rotating disks have been seen observationally is at redshifts $z=2-3$ (Genzel et al. 2006; Glazebrook 2013), and this has required deep spectroscopy on $10 \mathrm{~m}$ class telescopes. At higher redshifts, galaxies in published samples are very compact, with half-light radii $\sim 0.5 \mathrm{kpc}$ at $z=8$ (Oesch et al. 2010; Holwerda et al. 2014). The fraction of morphologically disturbed galaxies is also known to be high in deep fields (Menanteau et al. 2006). The volumes probed at redshifts $z>3$ and particularly the redshifts at the current frontier, $z=8-10$, are very small, however, with a sky area of $11 \operatorname{arcmin}^{2}$ accessible to the Hubble Extreme Deep Field (Illingworth et al. 2013), for example. This has meant that probing the highest-luminosity and largest galaxies has not been possible so far. It is not surprising that observational studies of the structure and morphologies of the highest $z$ galaxies have not revealed large disk galaxies, whether they exist or not.

Theoretically, the situation is similar-it is not known what the prevailing cold dark matter (CDM) model predicts for the most massive galaxies at redshifts $z \geqslant 8$. Numerical simulations used to make predictions have so far (Jaacks et al. 2012; Cen \& Kimm 2014) been limited to volumes 40-100 times smaller than our present work, or else zoomed simulations concentrating on individual galaxies (Pawlik et al. 2011) or overdensities (Yajima et al. 2014). Intriguingly, a dominant fraction of the early-forming (dwarf-mass) galaxies simulated so far show evidence of disklike structure (Pawlik et al. 2011; Romano-Díaz et al. 2011). It is of prime importance to reach the regime of large galaxies as the early formation of luminous or massive objects (such as first bright quasars and most massive galaxy clusters) is one of the most potent tests of models of structure formation in hierarchical cosmology. In order to do so and to simulate a statistically useful sample of galaxies, it is necessary to model a volume of the order of several hundred megaparsecs on a side. This must be done with a high enough mass and force resolution to allow studies of structure and morphology (see, e.g., Governato et al. 2007). We have been able to reach this regime with the BlueTides run, a cosmological hydrodynamic simulation that we have just completed, and we describe the first results below. 


\section{THE BLUETIDES SIMULATION}

BlueTides was carried out using the Smoothed Particle Hydrodynamics code MP-Gadget (see Springel 2005; Di Matteo et al. 2012 for earlier versions of the code) with $2 \times 7040^{3}$ (0.69 trillion) particles on 648,000 Cray XE compute cores of the Blue Waters system at the National Center for Supercomputing Applications. The simulation evolved a cube of side length 400 comoving $\mathrm{Mpch}^{-1}$ to redshift $z=8$, and it is the largest cosmological hydrodynamic simulation yet carried out in terms of memory usage by an order of magnitude (Di Matteo et al. 2012). The supernova feedback in BlueTides is modeled after Okamoto et al. (2010), with the wind feedback parameter $\kappa_{w}=3.7$. This model has been shown to reproduce several key properties of low-redshift galaxies (Vogelsberger et al. 2014). We also include the effect of supermassive black holes, primordial and metal cooling, H2 molecular cloud, and a non-uniform ionization background: we refer to Feng et al. (2015) for the parameters and other aspects of the simulation in more detail. We also show there that the global star formation rate (SFR) predicted is consistent with current observations at redshifts $z>8$. The volume simulated is approximately 300 times larger than the largest observational survey at these redshifts (Trenti et al. 2011).

Galaxies were selected from the raw particle data (47 TB per output time) using a friends-of-friends algorithm at a range of redshifts from $z=8-13$. The mass resolution of the simulation is $1.72 \times 10^{7} M_{\odot}$ per dark matter particle so that at redshift $z=8,36$ million galaxies were found containing 64 dark matter particles or more, above a mass threshold of $10^{9} M_{\odot}$. The BlueTides galaxy luminosity functions at the redshifts of interest here are fully consistent with current observations, which is shown in Feng et al. (2015). In the present case, we are most interested in the most massive galaxies with at least $10^{4}$ stellar particles, which contain enough particles to study their rotational support. The corresponding stellar mass is $0.7 \times 10^{10} M_{\odot}$ or more.

We have made images of all galaxies present in the simulation by adaptively smoothing the star particle distribution to reveal as much structure as possible given the resolution available. We have also used the SFR of the gas particles in the simulation to make other images. The SFR can be related to the rest-frame UV $(150 \mathrm{~nm})$ luminosity. The UV luminosity is sensitive to the presence of dust, and there is evidence that the highest-luminosity early galaxies are significantly obscured (Wilkins et al. 2013; Cen \& Kimm 2014). We account for this dust attenuation using a screening model (Joung et al. 2009). This simple model assumes that the dust attenuation $A_{\mathrm{UV}}$ in a galaxy pixel is proportional to the metal-mass density in that pixel. There is only one free parameter, which we normalize by setting $A_{\mathrm{UV}}=1$ for observed $M_{\mathrm{UV}}=-21$ galaxies (Wilkins et al. 2013). We have taken this approach, rather than performing a more detailed radiative transfer simulation, because the current major uncertainties lie in the dust content and attenuation curve (Wilkins et al. 2013).

\section{GALAXY MORPHOLOGY}

We turn directly to the morphology of the most massive $\left(M_{\text {stellar }} \geqslant 0.7 \times 10^{10} M_{\odot}\right)$ galaxies. We compute the inertia tensor of the star particles and use these to plot views of each galaxy parallel and perpendicular to the major axis. Studying them reveals that a significant fraction are visually disklike and surprisingly regular in shape.

In Figure 1, we show images of 10 disk galaxies in the simulation at $z=8$, both side-on and face-on for each. These are among those kinematically selected to be disks (see below). We can see that the disk components extend to a diameter of $\sim 4 \mathrm{kpc}$, with a median half-light radii $\sim 0.6 \mathrm{kpc}$ for those shown. Although the $z=8$ BlueTides disk galaxies have masses comparable to the Milky Way, their sizes are significantly smaller by a factor of $\sim 5$. The disk galaxies also have mean gas fractions of $\sim 70 \%$ at $z=8$. The typical stellar mass/halo mass ratio of these systems is a few percent $(\sim 5 \%$, estimated from friend-of-friend halos). This is similar to systems at $z=0$, where the stellar-to-halo ratio peaks at $4 \%$ for halos in the same mass range (Guo et al. 2010). The SFRs of these galaxies are $\sim 100 M_{\odot} \mathrm{yr}^{-1}$ orders of magnitude larger than the main-sequence galaxies of similar stellar mass at low $z$ (Whitaker et al. 2012). The stellar populations in the central regions of each galaxy are significantly older than the outer disk, with a proportion of stars that formed as early as $z=13$, i.e., age $300 \mathrm{Myr}$ at $z=8$.

These disk galaxies host supermassive black holes, with black hole masses ranging from a few times $10^{6}$ to a few times $10^{8} h^{-1} M_{\odot}$. Interestingly, the host galaxy of the most massive black hole $\left(3 \times 10^{8} h^{-1} M_{\odot}\right)$ has a much more dominant spheroidal component $(D / T=0.29)$ than typical galaxies with similar masses $(D / T>0.5)$. We plan to investigate the role of central black holes in the formation of disks in the near future.

Kinematic decomposition of the galaxies lets us know if the disklike structures seen in Figure 1 are rapidly rotating. We have used a standard technique (Governato et al. 2007) to determine the fraction of stars in each galaxy that are on planar circular orbits and that are associated with a bulge. We note that this technique is not sensitive to the clumpiness of disks. At redshift $z=8$, we find that $70 \%$ of galaxies above a mass of $10^{10} M_{\odot}$ are kinematically classified as disks (using the standard threshold of disk stars to total stars $(D / T)$ ratio of 0.2 (Governato et al. 2007). This percentage of disks changes with redshift as shown in Figure 2. In all cases, it is much higher than the fraction of observed galaxies of comparable mass that are seen to be disklike at $z=0,0.14 \pm 0.03$ (Buitrago et al. 2013).

We note that the majority of gas-rich disks seen at high redshifts accessible so far to spectroscopy $(z=2-3)$ are clumpy, turbulent, and thick (Förster Schreiber et al. 2009; Genzel et al. 2006 is an exception), and it is natural to expect this to be the case with the BlueTides massive disks, which have high gas fractions. A relevant statistic we have computed is the ratio of circular velocity $V$ to vertical velocity dispersion $\sigma$ of our disk galaxies. We have computed this $V / \sigma$ ratio using information from the disk star particles and plot a histogram of the results in Figure 3. We can see that the values range from $V / \sigma=4-6$, with a mean value of $4.98 \pm 0.04$ measured from all redshifts. The galaxies are therefore definitely disks, having $V / \sigma$ well above the $0-1$ expected for non-disk galaxies. This supports our conclusions made using the $D / T$ ratio above. The $V / \sigma$ values are lower than the $V / \sigma \sim 10$ expected for thin spiral galaxies seen at $z=0$ and instead are consistent with gasrich turbulent disks from earlier times (Förster Schreiber et al. 2009). Although this is reasonable, confirmation of this 


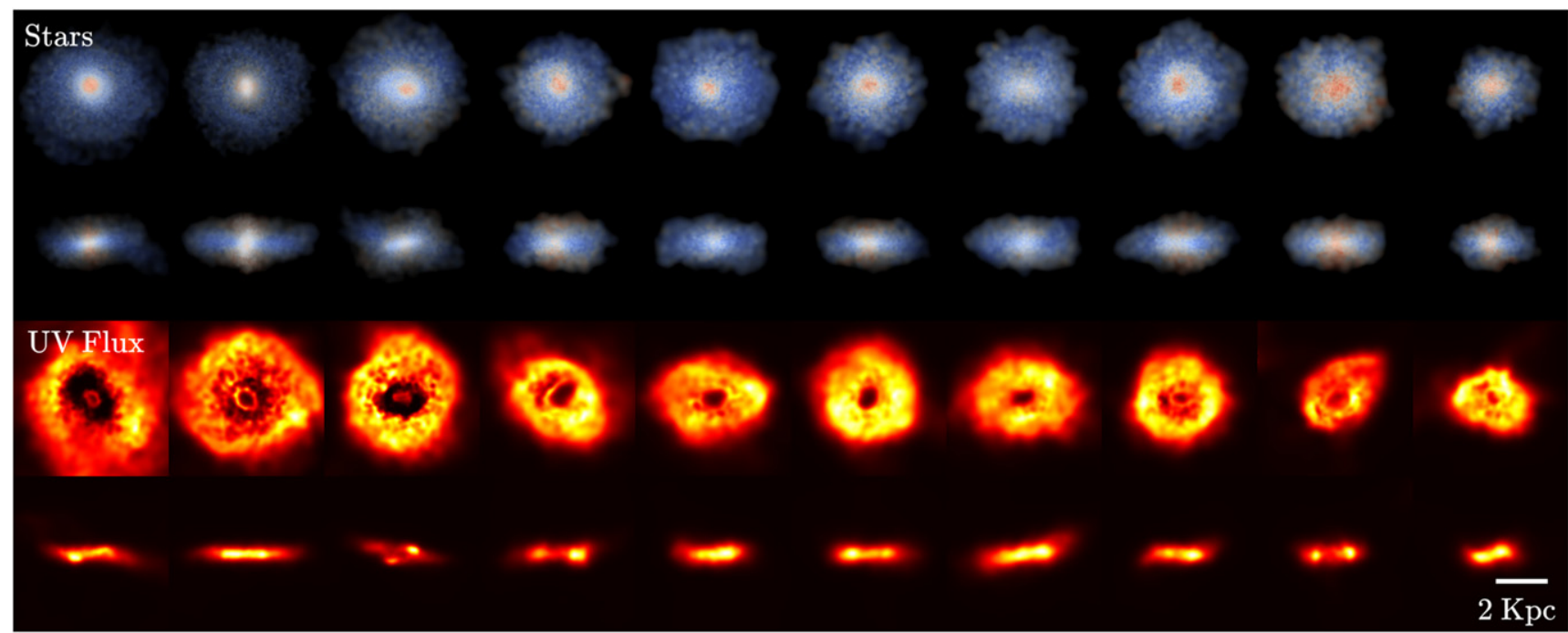

Figure 1. Sample of disk galaxies selected from the BlueTides simulation at redshift $z=8$. We show galaxies identified kinematically to be rotating disks. For each galaxy, we show a face-on and side-on view. The colors of the top two rows represent the stellar surface density coded by the stellar age (blue to red: $0 \sim 300 \mathrm{Myr}$ ) face-on and side-on. The colors of the bottom two rows represent the star formation surface density (normalized to $0 \sim 1$ ). The face-on images have included the effect of dust extinction. The darker central regions of the disks are mostly caused by the consumption of gas to form bulge stars. There is also an obscuration effect from the higher metallicity (and therefore dust content) of the central regions.

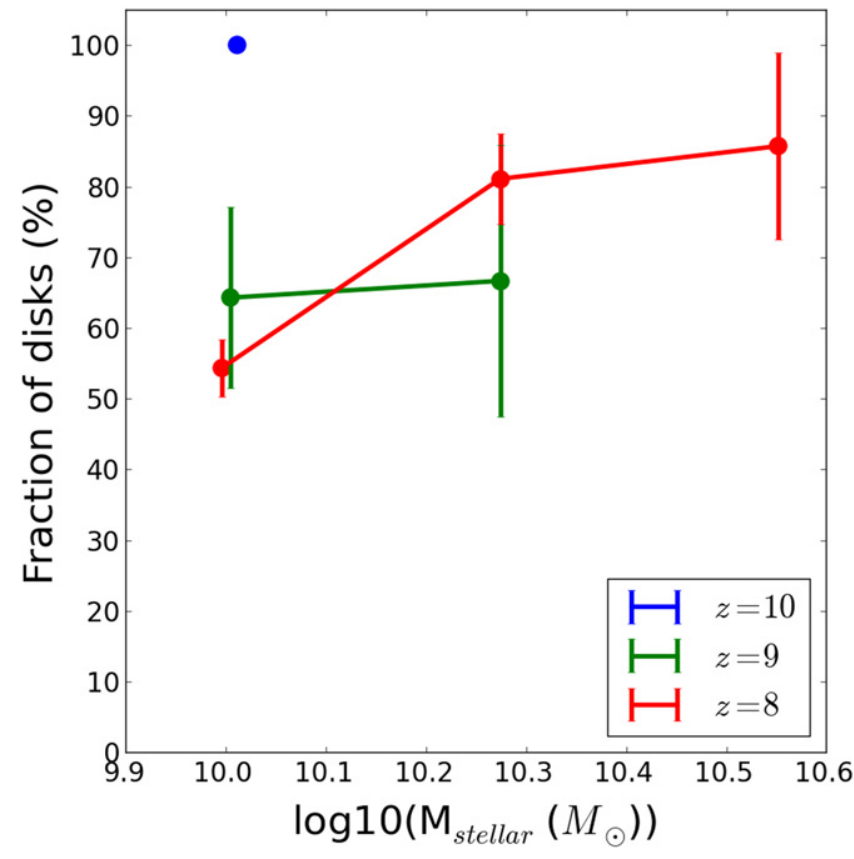

Figure 2. Fraction of galaxies in the BlueTides simulation kinematically classified as disks. Results are shown as a function of galaxy stellar mass and at three different redshifts.

aspect awaits future higher-resolution resimulations that are able to resolve the fine vertical structure of the disks.

\section{HALF-LIGHT RADIUS}

A number of recent studies (Oesch et al. 2010; Ono et al. 2013; Holwerda et al. 2014; Kawamata et al. 2014; Shibuya et al. 2015) have begun to make measurements of galaxy structure and in particular sizes at the highest redshifts. As the observational volumes are small, the magnitude range of observed samples varies from $M_{\mathrm{UV}}=-21$ to $M_{\mathrm{UV}}=-18$. We can therefore compare BlueTides galaxies with these measurements, bearing in mind that the brightest galaxies in the

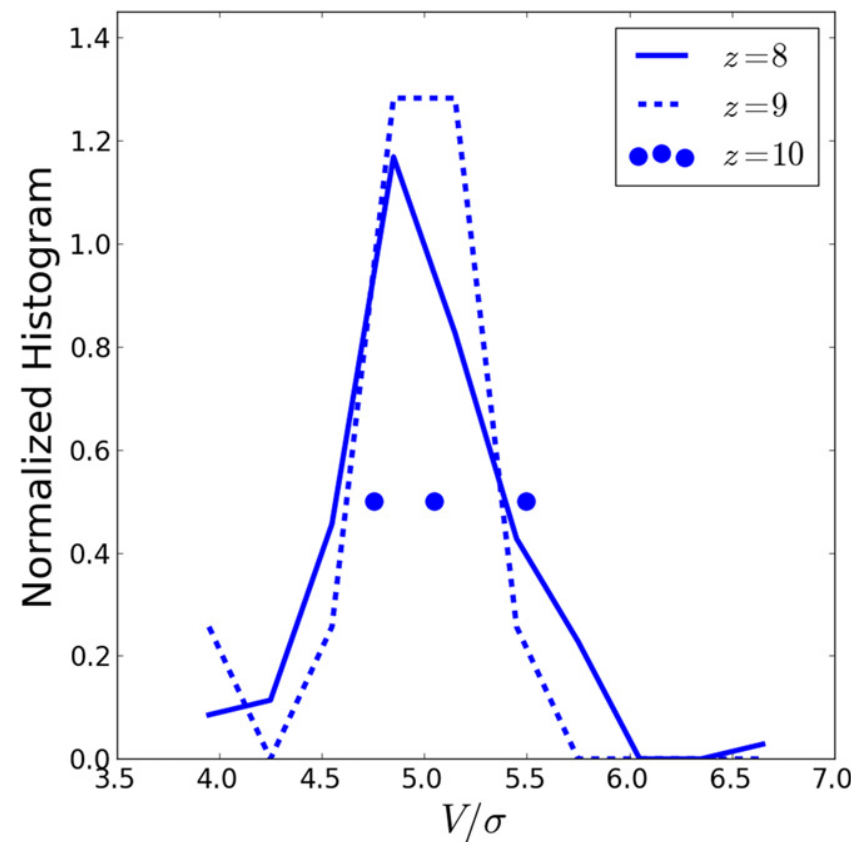

Figure 3. Disk galaxy kinematics in BlueTides characterized by the ratio of circular velocity $V$ to vertical velocity dispersion $\sigma$. We show histograms of results for this $V / \sigma$ ratio computed using star particles in the disks of galaxies selected to be disks from their $D / T$ ratio. Data on redshifts $z=8$ and $z=9$ are shown.

simulation are much rarer than can be observed in current surveys. Using the standard observational algorithm SEXtractor, ${ }^{5}$ we measure the half-light radius $\left(r_{1 / 2}\right)$ of each galaxy. This is shown in Figure 4, where we compare our redshift $z=8$ results with observational data from Kawamata et al. (2014), Holwerda et al. (2014), Curtis-Lake et al. (2014), and Shibuya et al. (2015). We can see that the current observations cover the lower end of the magnitude range and are in the same realm as

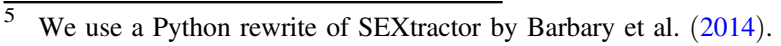




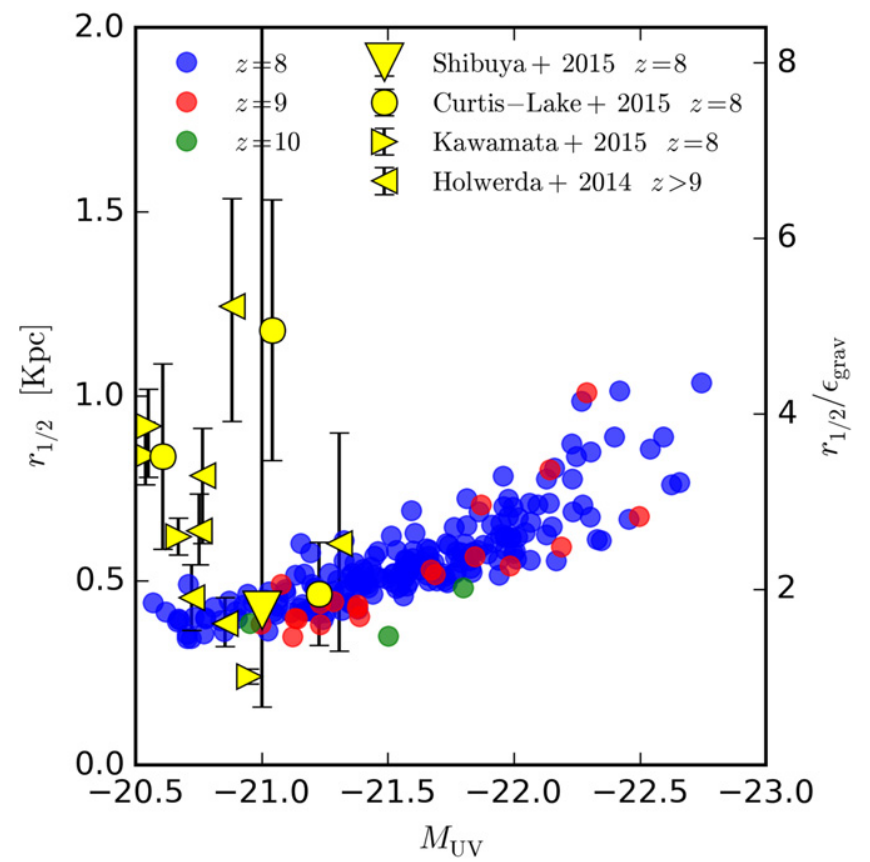

Figure 4. Galaxy half-light radii at redshift $z=8,9,10$ in the BlueTides simulation (including disks and non-disks) and in the Hubble Space Telescope observations of Holwerda et al. (2014), Kawamata et al. (2014), Curtis-Lake et al. (2014), and most recently Shibuya et al. (2015). Colored symbols: galaxies in BlueTides simulation; we include galaxies with a stellar mass $>5 \times 10^{9} h^{-1} M_{\odot}$ as upward wedges: Holwerda et al. (2014) $z \geqslant 9$ samples; downward wedges: Kawamata et al. (2014) $z=8$ samples. Circles: CurtisLake et al. (2014) $z=8$ undisturbed samples $M_{\mathrm{UV}}>-20.5$, with uncertainty estimated from their Figure 1. The half-light radius in BlueTides is measured from the face-on images. The right axis is in units of the force resolution $\epsilon_{\text {gravi }}=0.24 \mathrm{Kpc}$ at $z=8$.

(but not a good match to) our simulation results, with a mean $r_{1 / 2} \sim 0.6$ for $M_{\mathrm{UV}} \sim-20.5 \rightarrow-21.5$. Galaxies are therefore extremely compact at these high redshifts. The galaxy sizes in the simulation do appear to be on the low end compared to observations, something which has also been seen at $z=3$ (Joung et al. 2009) and could be a sign of interesting physics. It has also been shown, however (Curtis-Lake et al. 2014), that selection effects, flux cuts, and choice of size measurement algorithms can affect measured galaxy sizes by factors of two or more.

The half-light radius of the faintest samples $(\sim 0.5 \mathrm{Kpc})$ is only a factor of two of the gravitational smoothing length $(0.24 \mathrm{Kpc}$ at $z=8)$, which could also affect our results. In future work, we will explore these effects in more detail with mock observations. Interestingly, the simulation predicts a positive size-luminosity correlation.

Analytic models can be used to predict how disk sizes scale with redshift. For example, the simplest assumption is that the half-light radii scales with virial radius, which has the following redshift dependence:

$$
r_{\mathrm{vir}} \propto H(z)^{-1} \propto(1+z)^{-1.5}
$$

for constant halo circular velocity (e.g., Mo et al. 1998). Using this relationship to extrapolate the disk sizes from the GEMS observational sample analyzed in Somerville et al. (2008; in which the median $r_{d}$ for galaxies with $m_{*}=10^{10} M_{\odot}$ at $z=1.05$ is $2.1 \mathrm{Kpc}$ ), this simplest model predicts that disks of this mass should have $r_{d}=0.23 \mathrm{kpc}$ at $z=8$ and $r_{d}=0.17$ $\mathrm{kpc}$ at $z=10$. This is somewhat lower than disks of similar stellar mass in our simulation that have half-light radii about twice this value (see Figure 4-these correspond to the least luminous disks plotted). Somerville et al. (2008) have made more sophisticated models that include evolution in the internal structure of dark halos and adiabatic contraction, both of which mean that the trend of size evolution with redshift is expected to be weaker than in the simplest models (also supported by observations, e.g., Shibuya et al. 2015) and that therefore appear to describe our simulated results better.

\section{CONCLUSIONS}

At redshifts $z=8-10$, the universe is about 1000 times denser than the present day. In these very different physical conditions and in the very short time available, massive disk galaxies are able to form in the CDM model. They share many visual and kinematic characteristics with those that are seen when the universe was 20 times older. This means that an image one might have of the early universe containing only small protogalaxies, merging clumps, and irregular structures is not appropriate.

An immediate question we can ask is how these disks did in fact form in the simulation. We have tracked particles in the most massive galaxies (including those plotted in Figure 1) back in time to visually assess their formation history. We have found that of the most massive 20 galaxies at redshift $z=8$, only one was the product of an obvious major merger between similarly sized progenitor galaxies. A popular picture of gasrich major mergers producing some disks (Springel \& Hernquist 2005) therefore does not appear to be relevant here.

Even though the Lagrangian volumes (Oñorbe et al. 2014) and halos (Rossi et al. 2011) at the rare peaks associated with these galaxies are likely more spherical, the distribution of matter around galaxies is known to be highly anisotropic in the CDM model, and the infall of gas cannot proceed quasispherically as in the classical picture of galaxy formation (Fall \& Efstathiou 1980). Instead, smooth infall and accretion build up the disks, with cold gas arriving along the directions of nearby filaments in the density distribution (Romano-Díaz et al. 2014).

These massive disks will be fascinating objects to study with next-generation telescopes. The WFIRST satellite (Spergel et al. 2015) will have a field of view 200 times that of the WFC3 instrument on HST, making a planned deep $(J=26.7)$ sky survey covering $2000 \mathrm{deg}^{2}$ possible. Using the luminosity function in BlueTides and its evolution, we predict that this survey should find $\sim 8000$ disk galaxies of the type we have kinematically identified as massive disks, about one per field of view on average. This compares to a probability of 0.3 for finding a single one of these objects in the current largest-area HST survey (BoRG; Trenti et al. 2011). The rotation curves will be exciting targets for $30 \mathrm{~m}$ class ground-based telescopes. Forming from lightly enriched material, these primeval disk galaxies are special objects. Observing them and comparing them with simulations and analytic theory should enable us to make inferences about dark matter, the role of angular momentum, and the fundamental principles of galaxy formation (such as tidal torques and smooth accretion of unenriched material) that are not possible in the later, more complex universe. 
We acknowledge funding from NSF OCI-0749212 and NSF AST-1009781. The BlueTides simulation was run on BlueWaters supercomputer at the National Center for Supercomputing Applications.

\section{REFERENCES}

Barbary, K., Boone, K., \& Deil, C. 2014, SEP: Python and C Library for Source Extraction and Photometry, Zenodo, doi:10.5281/zenodo.15669

Buitrago, F., Trujillo, I., Conselice, C. J., \& Häußler, B. 2013, MNRAS, 428,1460

Cen, R., \& Kimm, T. 2014, ApJ, 782, 32

Curtis-Lake, E., McLure, R. J., Dunlop, J. S., et al. 2014, MNRAS, submitted (arXiv:1409.1832)

Di Matteo, T., Khandai, N., DeGraf, C., et al. 2012, ApJL, 745, L29

Eggen, O. J., Lynden-Bell, D., \& Sandage, A. R. 1962, ApJ, 136, 748

Fall, S. M., \& Efstathiou, G. 1980, MNRAS, 193, 189

Feng, Y., di Matteo, T., Croft, R. A. C., et al. 2015, MNRAS, submitted

Förster Schreiber, N. M., Genzel, R., Bouché, N., et al. 2009, ApJ, 706, 1364

Genzel, R., Tacconi, L. J., Eisenhauer, F., et al. 2006, Natur, 442, 786

Glazebrook, K. 2013, PASA, 30, 56

Governato, F., Willman, B., Mayer, L., et al. 2007, MNRAS, 374, 1479

Guo, Q., White, S., Li, C., \& Boylan-Kolchin, M. 2010, MNRAS, 404, 111

Holwerda, B. W., Bouwens, R., Oesch, P., et al. 2014, ApJ, submitted (arXiv:1406.1180)

Illingworth, G. D., Magee, D., Oesch, P. A., et al. 2013, ApJS, 209, 6

Jaacks, J., Choi, J.-H., Nagamine, K., Thompson, R., \& Varghese, S. 2012, MNRAS, 420, 1606

Joung, M. R., Cen, R., \& Bryan, G. L. 2009, ApJL, 692, L1

Kawamata, R., Ishigaki, M., Shimasaku, K., Oguri, M., \& Ouchi, M. 2014, ApJ, in press (arXiv:1410.1535)
Menanteau, F., Ford, H. C., Motta, V., et al. 2006, AJ, 131, 208

Mo, H. J., Mao, S., \& White, S. D. M. 1998, MNRAS, 295, 319

Oesch, P. A., Bouwens, R. J., Carollo, C. M., et al. 2010, ApJL, 709, L21

Okamoto, T., Frenk, C. S., Jenkins, A., \& Theuns, T. 2010, MNRAS, 406, 208

Ono, Y., Ouchi, M., Curtis-Lake, E., et al. 2013, ApJ, 777, 155

Oñorbe, J., Garrison-Kimmel, S., Maller, A. H., et al. 2014, MNRAS, 437, 1894

Pawlik, A. H., Milosavljević, M., \& Bromm, V. 2011, ApJ, 731, 54

Press, W. H., \& Schechter, P. 1974, ApJ, 187, 425

Romano-Díaz, E., Choi, J.-H., Shlosman, I., \& Trenti, M. 2011, ApJL, 738, L19

Romano-Díaz, E., Shlosman, I., Choi, J.-H., \& Sadoun, R. 2014, ApJL, 790, L32

Rossi, G., Sheth, R. K., \& Tormen, G. 2011, MNRAS, 416, 248

Shibuya, T., Ouchi, M., \& Harikane, Y. 2015, arXiv:1503.07481

Shimizu, I., Inoue, A. K., Okamoto, T., \& Yoshida, N. 2014, MNRAS, 440,731

Silk, J., \& Mamon, G. A. 2012, RAA, 12, 917

Somerville, R. S., Hopkins, P. F., Cox, T. J., Robertson, B. E., \& Hernquist, L. 2008, MNRAS, 391, 481

Spergel, D., Gehrels, N., Baltay, C., et al. 2015, Wide-Field InfrarRed Survey Telescope-Astrophysics Focused Telescope Assets WFIRST-AFTA Rep., arXiv: 1503.03757

Springel, V. 2005, MNRAS, 364, 1105

Springel, V., \& Hernquist, L. 2005, ApJL, 622, L9

Trenti, M., Bradley, L. D., Stiavelli, M., et al. 2011, ApJL, 727, L39

Vogelsberger, M., Genel, S., Springel, V., et al. 2014, MNRAS, 444, 1518

Whitaker, K. E., van Dokkum, P. G., Brammer, G., \& Franx, M. 2012, ApJL, 754, L29

Wilkins, S. M., Coulton, W., Caruana, J., et al. 2013, MNRAS, 435, 2885

Yajima, H., Shlosman, I., Romano-Diaz, E., \& Nagamine, K. 2014, ApJ, in press (arXiv:1411.2626) 